INTERVENTIONAL CARDIOLOGY AND SURGERY

\title{
Predictors of outcome after percutaneous treatment for cardiogenic shock
}

\author{
A G C Sutton, P Finn, J A Hall, A A Harcombe, R A Wright, M A de Belder
}

Heart 2005;91:339-344. doi: 10.1136/hrt.2003.021691

See end of article for authors' affiliations

.....................

Correspondence to:

Dr Andrew G C Sutton,

Cardiothoracic Division,

The James Cook University

Hospital, Marton Road,

Middlesbrough TS4 3BW,

UK; Andrew.Sutton@

stees.nhs.uk

Accepted 15 April 2004
Objectives: To determine predictors of outcome after percutaneous coronary intervention (PCI) in patients with cardiogenic shock complicating acute myocardial infarction.

Methods: Retrospective analysis of a cohort of 113 patients undergoing emergency coronary angiography and attempted $\mathrm{PCl}$ for cardiogenic shock complicating acute myocardial infarction in a regional cardiothoracic unit.

Results: In-hospital mortality was 51\% (58 patients). Adverse outcome was associated with previous myocardial infarction, age over 70 years, cardiogenic shock complicating failure to respond to thrombolytic treatment (failed thrombolysis), and multivessel coronary artery disease. Multivariate logistic regression analysis showed that the first three factors were independent predictors of in-hospital death with odds ratios of 5.21 (95\% confidence interval (Cl) 1.85 to 14.69), 4.02 (95\% Cl 1.14 to 14.12), and 3.78 (95\% Cl 1.43 to 9.96$)$, respectively.

Conclusion: About $50 \%$ of patients with cardiogenic shock undergoing a strategy of urgent coronary angiography and $\mathrm{PCl}$ survive to hospital discharge. Survivors do well in the subsequent six months. Emergency $\mathrm{PCl}$ for cardiogenic shock reduces mortality from an expected $80 \%$ to about $50 \%$. Clinical features can help determine which patients are most likely to gain from urgent coronary angiography and attempted $\mathrm{PCl}$. Alternative strategies are needed to improve the outcome of patients who fare badly.
$\mathrm{C}$ ardiogenic shock occurs in $7-10 \%$ of patients after acute myocardial infarction (AMI). Untreated, it causes an early mortality of about $80 \%^{12}$ and is the leading cause of death among patients hospitalised with AMI.

The incidence of cardiogenic shock did not fall significantly during the thrombolytic era. There is some evidence to show that tissue plasminogen activator is more effective than streptokinase in the prevention of cardiogenic shock, ${ }^{2}$ but little evidence from randomised trials of thrombolysis in AMI to support the use of these agents when shock is present. ${ }^{3}$ Non-randomised studies have consistently suggested that emergency coronary angiography and revascularisation reduce mortality of patients with cardiogenic shock..$^{4-9}$

Data from randomised controlled trials have been lacking until the publication of the SHOCK (should we emergently revascularise occluded coronaries for cardiogenic shock?) trial. ${ }^{10}$ This trial randomly assigned patients with cardiogenic shock to an emergency revascularisation arm or an intensive medical treatment arm. Those in the emergency revascularisation arm fared better at six months than those in the medical arm. Patients in the medical arm also fared better than historical patients receiving standard medical treatment for cardiogenic shock. It is clear that some patients do well with an aggressive revascularisation strategy but many continue to do badly. We studied a consecutive series of patients treated with percutaneous coronary intervention (PCI) for cardiogenic shock caused by left ventricular dysfunction to determine whether clinical features could predict those who continue to do badly.

\section{METHODS}

We adopted a strategy of emergency angiography and attempted PCI for cardiogenic shock complicating AMI in 1995. This service was offered to all surrounding district general hospitals serving our unit. The aim was to offer emergency revascularisation within 12 hours of the onset of symptoms, although revascularisation was attempted in some patients later than this because of continuing chest pain. The service was offered around the clock. There was no requirement for measurement of cardiac filling pressures or cardiac output by invasive monitoring for the diagnosis to be made, but patients were required to fulfil the following criteria for cardiogenic shock as defined by the British Cardiovascular Intervention Society: blood pressure $<100 \mathrm{~mm} \mathrm{Hg}$; pulse > 100 beats/min; and the patient cool, clammy, or requiring inotropes, intra-aortic balloon pump (IABP), or cardiopulmonary support to assist the circulation. Measurement of urine output was not mandatory. After clinical evaluation, echocardiography and occasionally left ventriculography were used to identify patients with cardiogenic shock caused by papillary muscle rupture, interventricular septal defect, or cardiac rupture before PCI, and these patients were not included in the analysis. Patients for whom coronary artery bypass grafting (CABG) was considered the best initial revascularisation strategy were not included in the analysis.

Coronary angiography was performed without routine left ventriculography. The use of an IABP in the catheterisation laboratory was left to the discretion of the attending cardiologist, with insertion of the device either before or after PCI. The strategy was percutaneous intervention to the culprit vessel if this could be identified, with intervention to other critical coronary stenoses only attempted in the absence of early haemodynamic improvement. The aim was to keep the procedure as short as possible and minimise the contrast load. The use of adjunctive pharmacological treatment and

Abbreviations: $A M I$, acute myocardial infarction; $C A B G$, coronary artery bypass grafting; CAPTIM, comparison of angioplasty and prehospital thrombolysis in acute myocardial infarction; $\mathrm{Cl}$, confidence interval; GUSTO-I, global utilisation of streptokinase and tissue plasminogen activator for occluded coronary arteries; IABP, intra-aortic balloon pump; $\mathrm{PCl}$, percutaneous coronary intervention; SHOCK, should we emergently revascularise occluded coronaries for cardiogenic shock?; TIMI, thrombolysis in myocardial infarction 
Table 1 Patient characteristics of patients at the James Cook University Hospital (JCUH) and patients receiving $\mathrm{PCl}$ in the revascularisation arm of the SHOCK (should we emergently revascularise occluded coronaries for cardiogenic shock?) trial

\begin{tabular}{|c|c|c|}
\hline Characteristic & JCUH $(n=113)$ & $\mathrm{PCl}$ in $\mathrm{SHOCK}$ trial $^{17}(\mathrm{n}=82)$ \\
\hline Age range (years) & $26-79$ & NA \\
\hline Age (years) & $60.1(10.5)$ & $65(10)$ \\
\hline Men & $76(67 \%)$ & $62 \%$ \\
\hline Anterior Ml & $59(52 \%)$ & NA \\
\hline Non-anterior Ml & $54(48 \%)$ & NA \\
\hline Primary angioplasty & $41(36 \%)$ & NA \\
\hline Rescue angioplasty for failed thrombolysis & $47(42 \%)$ & Thrombolysis $49 \%$ \\
\hline Angioplasty for reinfarction & $25(22 \%)$ & NA \\
\hline Mean chest pain onset to $\mathrm{PCl}$ (hours) & $7.4(5.9)$ & NA \\
\hline Median chest pain onset to $\mathrm{PCI}$ (hours) & $5.8(3.4-10)$ & $11.0(6.1-19.5)$ \\
\hline Transferred patients & $71(63 \%)$ & $60 \%$ \\
\hline Local patients & $42(37 \%)$ & NA \\
\hline Previous MI & $34(30 \%)$ & $23 \%$ \\
\hline Diabetes & $21(19 \%)$ & $25 \%$ \\
\hline Previous CABG & $3(2.7 \%)$ & $4 \%$ \\
\hline Hypertension & $23(20 \%)$ & NA \\
\hline Unknown & $5(4.4 \%)$ & NA \\
\hline Current or former smoker & $80(71 \%)$ & NA \\
\hline Unknown & $6(5.3 \%)$ & NA \\
\hline Hyperlipidaemia* & $70(62 \%)$ & NA \\
\hline Unknown & $5(4.4 \%)$ & NA \\
\hline \multicolumn{3}{|c|}{$\begin{array}{l}\text { Data are mean (SD), median (interquartile range), or number (\%). } \\
\text { *History of hyperlipidaemia and taking cholesterol lowering medication, or random total cholesterol on admission } \\
\geqslant 5 \mathrm{mmol} / \mathrm{l} \text {. } \\
\text { CABG, coronary artery bypass graft; MI, myocardial infarction; } \mathrm{NA} \text {, not available; } \mathrm{PCl} \text {, percutaneous coronary } \\
\text { intervention. } \\
\text { Unknown data relate mostly to patients who were too sick to give this information before } \mathrm{PCl} \text { and for whom no } \\
\text { evidence was subsequently found. }\end{array}$} \\
\hline
\end{tabular}

other mechanical devices in the catheterisation laboratory was left to the discretion of the attending cardiologist.

Initially, unit guidelines were in place regarding the use of glycoprotein IIb/IIIa inhibitors following previous favourable experience with the selective use of this agent. ${ }^{11}$ These guidelines favoured the bailout use of this agent rather than planned use, especially in the context of cardiogenic shock after full dose thrombolytic, given the concern about bleeding risks. The indications for administration were: (1) the presence of intracoronary filling defects or haze after PCI, suggestive of thrombus; (2) slow flow after intracoronary stenting; (3) persistent dissection flaps; (4) suspected inlet or outlet dissection flaps after stenting; and (5) after long or multiple stenting, especially in the context of acute coronary syndromes or AMI. More recently, following additional trials of abciximab in primary PCI, abciximab has been used more liberally in patients who have not been treated with full dose thrombolytic.

Our stenting strategy changed over time, according to availability of trial evidence to support the use of stenting in patients with AMI. Initially, stents were used for suboptimal balloon angioplasty results, but more recently elective stenting has been the preferred strategy. If one or more coronary stents were deployed, ticlopidine $250 \mathrm{mg}$ twice daily after an initial immediate dose of $500 \mathrm{mg}$ or clopidogrel $75 \mathrm{mg}$ once daily after an initial dose of $300 \mathrm{mg}$ was administered. Patients were subsequently nursed on the coronary care unit or on the cardiac intensive care unit if they were being ventilated.

\section{Statistical methods}

Data were collected prospectively with retrospective review of the medical notes to obtain missing information. All data were retrospectively analysed with the assistance of SPSS version 10.0 (SPSS Inc, Chicago, Illinois, USA). To compare mortality across single variables, the $\chi^{2}$ test $(2 \times 2$ contingency tables) was used. Multivariate logistic regression analysis (forward stepwise method) was used to identify the combination of single variables that were independent predictors of death; significant variables analysed are reported with their respective odds ratios and 95\% confidence intervals.

\section{RESULTS}

Between 1995 and 2002, we performed emergency coronary angiography and attempted PCI in 113 patients with cardiogenic shock complicating AMI. There were 76 (67\%) men and 37 (33\%) women; their mean (SD) age was 60.1 (10.5) years and ranged from 26-79 years. Table 1 shows their clinical characteristics. Coronary angiography showed multivessel disease in $79 \%$ and the culprit vessel was the left main stem in six patients. Nineteen patients (17\%) were ventilated in the catheterisation laboratory and in 11 patients $(9.7 \%)$ the last remaining vessel was occluded. Primary angioplasty (that is, angioplasty without antecedent thrombolytic treatment) was performed in 31 patients, rescue angioplasty for failure to respond to a thrombolytic in 47 patients, and angioplasty for cardiogenic shock complicating reinfarction in 25 patients. All patients with reinfarction had received thrombolytic treatment for the initial presentation with ST elevation myocardial infarction and three had received a second dose of a thrombolytic when reinfarction occurred.

The infarct related vessel, when clearly identifiable, was occluded in $83 \%$ (table 2). Eighty two patients (73\%) underwent technically successful PCI, defined by a patent vessel with TIMI (thrombolysis in myocardial infarction) 2 or 3 flow, ${ }^{12}<50 \%$ residual stenosis, no emergency CABG from the catheterisation laboratory, and successful transfer to the coronary care unit or cardiac intensive care unit. The remaining 31 patients $(27.4 \%)$ had an unsuccessful procedure. Of these, 11 died in the catheterisation laboratory and five underwent emergency CABG, of whom four died during the index admission. The following adjunctive treatments were used in the catheterisation laboratory: IABP in $91 \%$; inotropes in 50\%; stent(s) in $48 \%$; abciximab in $25 \%$; and a 
Table 2 Angiographic features and procedures

\begin{tabular}{|c|c|c|}
\hline & JCUH $(n=113)$ & $\mathrm{PCl}$ in SHOCK trial ${ }^{17}(n=82)$ \\
\hline Multivessel coronary disease & $89(79 \%)$ & $81 \%$ \\
\hline LMS disease $>50 \%$ & $17(15 \%)$ & $14 \%$ \\
\hline Single vessel disease & $24(21 \%)$ & $19 \%$ \\
\hline \multicolumn{3}{|l|}{ Culprit vessel } \\
\hline LAD & $58(51 \%)$ & $50 \%$ \\
\hline RCA & $33(29 \%)$ & $33 \%$ \\
\hline Circumflex & $12(11 \%)$ & $9 \%$ \\
\hline LMS & $6(5.3 \%)$ & $5 \%$ \\
\hline Unknown & $1(0.9 \%)$ & NA \\
\hline Saphenous vein graft & $3(2.7 \%)$ & $4 \%$ \\
\hline Total occlusion in culprit vessel & $94(83 \%)$ & TIMI 0 or $1,62 \%$ \\
\hline Culprit vessel last remaining vessel & $11(9.7 \%)$ & NA \\
\hline Stent(s) & $54(48 \%)$ & $34 \%$ \\
\hline IABP & $103(91 \%)$ & $89 \%$ \\
\hline Abciximab & $28(25 \%)$ & Gp llb/Illa inhibitor, 32\% \\
\hline Ventilation & $19(17 \%)$ & $84 \%$ \\
\hline Pacing wire & $21(19 \%)$ & NA \\
\hline Inotropic support & $57(50 \%)$ & $100 \%$ \\
\hline $\mathrm{PCl}$ success & $82(73 \%)$ & $76 \%$ \\
\hline
\end{tabular}

temporary pacing wire in $19 \%$. One patient had a successful PCI procedure and underwent urgent planned CABG the next day for severe triple vessel disease.

In-hospital mortality was $51 \%(\mathrm{n}=58)$. A failed laboratory procedure was associated with an in-hospital mortality of $83.9 \%$ and a successful laboratory outcome, by the above definition, with an in-hospital mortality of $39.0 \%$. Increased age $(\geqslant 70$ years), previous myocardial infarction, failed thrombolysis, and multivessel coronary artery disease were all associated with excess mortality compared with patients without these features (table 3 ), although only the first three factors were observed to be independent predictors of death (table 4). All three patients with previous CABG died.

The time from onset of major symptoms to coronary intervention could be determined for 103 patients (91\%). Mean (SD) time was 7.4 (5.9) hours and median time (interquartile range) was $5.8(3.4-10)$ hours. However, in this series, in-hospital outcome was not clearly related to the time from the onset of symptoms to arrival in the cardiac catheterisation laboratory (table 3 ).

Three patients who underwent successful PCI were readmitted electively within the next three months for CABG. Patients who survived to hospital discharge were likely to be alive and well six months after presentation. Survival at six months was $45 \%$ (fig 1 ).

\section{DISCUSSION}

Results of previous studies vary, in part due to differences in the definition of cardiogenic shock. In the GUSTO-I (global utilisation of streptokinase and tissue plasminogen activator for occluded coronary arteries) study, cardiogenic shock was defined by a systolic blood pressure of $\leqslant 90 \mathrm{~mm} \mathrm{Hg}$ for $\geqslant 1$ hour, unresponsive to fluid challenge, thought to be secondary to cardiac dysfunction, and associated with signs of hypoperfusion or cardiac index $\leqslant 2 \mathrm{l} / \mathrm{min} / \mathrm{m}^{2}{ }^{13}$ Other criteria are oliguria $(<20 \mathrm{ml} / \mathrm{h})$, cerebral obtundation, and

\begin{tabular}{|c|c|c|c|}
\hline Factor & Mortality & $\mathrm{p}$ Value & Test details \\
\hline Previous MI & & $<0.001$ & $\chi^{2}=12.31, d f=1$ \\
\hline $\begin{array}{l}\text { No } \\
\text { Yes }\end{array}$ & $32(41 \%)$ & & \\
\hline $\begin{array}{l}\text { Yes } \\
\text { Age (years) }\end{array}$ & $26(77 \%)$ & 0.02 & $\chi^{2}=5.49, \mathrm{df}=1$ \\
\hline$<70$ & $40(46 \%)$ & & \\
\hline$\geqslant 70$ & $18(72 \%)$ & & \\
\hline Failed reperfusion & & $<0.001$ & $\chi^{2}=14.22, d f=1$ \\
\hline No & $24(36 \%)$ & & \\
\hline Yes & $34(72 \%)$ & & \\
\hline Disease & & 0.01 & $\chi^{2} 5.99, \mathrm{df}=1$ \\
\hline $\begin{array}{l}\text { Single vessel } \\
\text { Multivessel }\end{array}$ & $\begin{array}{l}7(29 \%) \\
51(57 \%)\end{array}$ & & \\
\hline Sex & 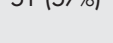 & 0.99 & $\chi^{2}<0.01, d f=1$ \\
\hline Women & 19 (51\%) & & \\
\hline Men & $39(51 \%)$ & & \\
\hline Smoking $(n=107)^{*}$ & & 0.61 & $\chi^{2}=0.26, d f=1$ \\
\hline No & $32(52 \%)$ & & \\
\hline Yes & $21(47 \%)$ & & \\
\hline Diabetes $(n=110)^{*}$ & & 0.81 & $\chi^{2}=0.06, d f=1$ \\
\hline No & $45(51 \%)$ & & \\
\hline Yes & $10(48 \%)$ & & \\
\hline Time to intervention $(n=103)^{*}$ & & 0.32 & $\chi^{2}=2.28, d f=2$ \\
\hline$\leqslant 6$ hours & $23(43 \%)$ & & \\
\hline 6-12 hours & 19 (59\%) & & \\
\hline$>12$ hours & $8(47 \%)$ & & \\
\hline
\end{tabular}


Table 4 In-hospital mortality: multivariate logistic regression analysis $(n=97)$

\begin{tabular}{lll}
\hline Predictive factors & OR $(95 \% \mathrm{Cl})$ & $\mathbf{p ~ V a l u e ~}$ \\
\hline Previous MI & $5.21(1.85$ to 14.69$)$ & $<0.01$ \\
Age $\geqslant 70$ & $4.02(1.14$ to 14.12$)$ & 0.03 \\
Failed reperfusion & 3.781 .43 to 9.96$)$ & 0.01 \\
\hline \multicolumn{2}{l}{$\mathrm{Cl}$, confidence interval; OR, odds ratio. } &
\end{tabular}

hypotension despite a pulmonary arterial wedge pressure of $18-20 \mathrm{~mm} \mathrm{Hg}$. Systemic vascular resistance is usually high in patients with cardiogenic shock, although this is a not an absolute requirement for the diagnosis. ${ }^{14}$ On occasion, cardiogenic shock is caused by a mechanical problem such as a ruptured papillary muscle, mitral valve chord, interventricular septum, or left ventricular free wall. Under these circumstances, urgent surgery may be considered. Most commonly, however, cardiogenic shock complicating AMI is caused by extensive damage to the left ventricular myocardium.

The SHOCK study by Hochman and colleagues ${ }^{10}$ suggests that, firstly, an invasive strategy with early revascularisation is associated with a better long term outcome than continued medical treatment; and, secondly, intensive medical treatment (including ventilation when necessary, IABP, and late revascularisation) is associated with a better outcome than previously experienced with a conservative medical approach. The authors persisted with the trial over a five and a half year period, despite problems with slow recruitment and logistical difficulties in transferring critically ill patients, often with ventilatory support and IABP. An impressive 97\% of patients in the revascularisation arm underwent angiography and $87 \%$ were revascularised. Only $2.7 \%$ of patients in the intensive medical treatment arm crossed over to the revascularisation arm less than $\mathbf{5 4}$ hours after randomisation.

In the revascularisation group of the SHOCK study, the median time from randomisation to the first revascularisation attempt was 0.9 hours for patients undergoing PCI and 2.7 hours for those undergoing $C A B G$, with the median time from chest pain onset to randomisation being 11 hours. Unlike in our study, patients could be treated if cardiogenic shock began within 36 hours of the onset of myocardial infarction.

Mortality at 30 days was $46.7 \%$ in the revascularisation group and $56.0 \%$ in the medically treated group $(\mathrm{p}=0.11)$. As has been pointed out, ${ }^{15}$ the SHOCK trial was almost certainly underpowered for the primary end point of 30 day all cause mortality because of the exaggerated expectation that revascularisation would save 200 lives per 1000 patients treated. This would have been a $40 \%$ relative reduction in mortality if the medical group had an expected mortality of $50 \%$ or a $27 \%$ relative reduction if the expected mortality was $75 \%$ in the medical arm. Failure to obtain a significant early result is probably due to the lower than expected mortality in the intensely treated medical arm, which is probably explained by the frequent use of IABP in addition to thrombolysis and late revascularisation in this group. Nevertheless, the trial had a relative reduction in 30 day mortality of $17 \%$, still therapeutically significant and representing 93 lives saved per 1000 patients treated.

In the SHOCK study, mortality at six months (a secondary end point) was significantly lower in the revascularisation group $(50.3 \% \vee 63.1 \%, p=0.03)$, representing 128 lives saved per 1000 patients treated. At one year, mortality in the revascularisation arm was $53.3 \%$ versus $66.4 \%,{ }^{16}$ a $13.2 \%$ difference (14.1\% if eight patients with dissection, tamponade, or severe mitral regurgitation are excluded), representing 132 (or 141) lives saved per 1000 patients treated.

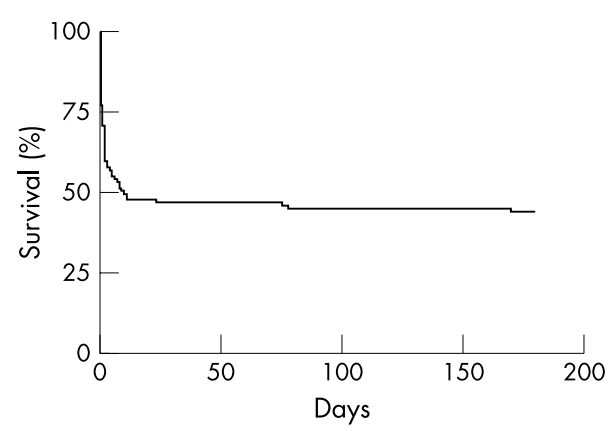

Figure 1 Six month survival.

Patient selection may explain the slight difference in results between our study and the SHOCK trial or registry. The SHOCK trial patients seem to have been a sicker group than ours, for a number of reasons: patients were required to have a systolic blood pressure $\leqslant 90 \mathrm{~mm} \mathrm{Hg}$ rather than $<100 \mathrm{~mm} \mathrm{Hg}$; the median (interquartile range) time from chest pain onset to randomisation in the revascularisation arm was 11.0 hours (5.9-19.4) compared with 5.8 hours (3.410 ) in our study; the patients were older (mean age 65.8 years $v 60.1$ years); more had an anterior infarct (63.6\% v 52\%); more were treated with inotropes or vasopressors $(99.3 \% v$ $50 \%)$; more were ventilated ( $88 \%$ v $17 \%)$, more were diabetic $(31.1 \% \vee 19 \%)$; and more had significant left main stem disease $(23.4 \% \vee 15 \%)$.

However, some of these differences are explained by our cohort consisting of patients deemed suitable for urgent PCI as the principal revascularisation method. Our cohort did not include patients with mechanical causes of cardiogenic shock, nor did it include patients for whom the best revascularisation strategy was insertion of an IABP followed by emergency surgery. In the SHOCK trial, $37.5 \%$ of the early revascularisation group underwent early CABG with a $59.5 \%$ 30 day survival rate, slightly higher than the PCI treated patients $(54 \%)$, even though the CABG group had more extensive coronary disease. It is not known whether patients with "surgical disease" fare better with immediate CABG or immediate culprit vessel PCI followed by delayed CABG.

Our patients are similar to the patients undergoing revascularisation by PCI in the SHOCK trial $(\mathrm{n}=82),{ }^{17}$ although younger and with shorter pain to revascularisation times (tables 2 and 3). The proportion of transferred patients in our series is similar to the proportion of patients receiving PCI in the SHOCK trial $(63 \% v 60 \%)$. PCI success rates were similar $(73 \% v 76 \%)$, with a similar definition of success in the SHOCK trial (residual stenosis $\leqslant 50 \%$, $\geqslant 20 \%$ reduction in stenosis, and TIMI 2 or 3 flow). In-hospital or 30 day mortality $(51 \% \vee 46 \%)$ is comparable. For patients with successful angioplasty, in-hospital mortality in our series was $39 \%$ versus $38 \% 30$ day mortality in the SHOCK trial's patients treated with PCI.

Interestingly, patients in the SHOCK PCI arm were ventilated much more often, but the outcomes were similar to our own experience. Although we cannot be certain, one explanation is that our patients were younger and presented earlier so that the requirement for ventilation was reduced. It is possible, however, that some patients may have fared better with earlier ventilation. We do not know whether planned multivessel PCI would have resulted in improved outcomes and there are few randomised data, certainly not in the context of cardiogenic shock, for guidance. At present, we favour a strategy of culprit vessel PCI, which minimises contrast load, reserving PCI to the non-culprit vessel(s) for lack of haemodynamic improvement. 
In our series, patients in shock undergoing rescue angioplasty had a particularly high mortality $(72 \% \vee 36 \%$ for those without prior thrombolysis). Administration of a thrombolytic before PCI did not affect survival in patients undergoing PCI for cardiogenic shock in the SHOCK trial registry, ${ }^{18}$ although the registry data as a whole do suggest that patients with cardiogenic shock should be considered for thrombolytic treatment if there is no option for revascularisation. $^{19}$ The reasons for the difference between our experience and the SHOCK trial PCI registry are unclear, but part of the explanation may be definition. We use the expression "rescue angioplasty" to describe angioplasty delivered for tightly defined failure of thrombolysis. ${ }^{20}$ It is unclear how many patients in the SHOCK registry or trial had cardiogenic shock accompanying a diagnosis of failed thrombolysis. However, $56.3 \%$ of all randomly assigned patients in SHOCK, $49.3 \%$ of those in the revascularisation arm and $49 \%$ of those in the revascularisation arm receiving PCI, had received a thrombolytic. Only $21 \%$ of those receiving PCI had TIMI 3 flow in the culprit vessel, so some patients did have cardiogenic shock complicating failed thrombolysis.

For some, the strategy of attempted emergency revascularisation remains controversial. There is an argument that the SHOCK trial failed to show benefit in the revascularisation arm, given that the incidence of the primary end point in the two groups was not significantly different. Conversely, others interpret the final results as being strongly supportive of an interventional strategy. It is highly improbable that further randomised trials of emergency revascularisation versus medical treatment will be performed, because of perceived ethical issues and slow recruitment. Even if one interpretation of the SHOCK trial is that revascularisation is not superior to intense medical management, this is of little help in determining management of cardiogenic shock in the UK, since the intense form of medical management used in this trial is also not widely practised in the UK. Medical staff in district general hospitals are not, in general, familiar with the use of an IABP, for example, and are reluctant to ventilate patients who are perceived to be dying. Therefore, we can either ignore the trial results, in which case patients with cardiogenic shock will continue to be managed on an ad hoc basis, or we can accept that attempted revascularisation appears to be the superior strategy with the proviso that certain groups of patients are more likely to benefit than others.

Our results add to the accumulating evidence to support intervention in cardiogenic shock. This strategy is particularly beneficial in certain groups of patients but appears to have little impact on the natural history of the condition in others. Our experience allows particular insights into the management of this uncommon but often fatal condition.

Firstly, patients with previous myocardial infarction and cardiogenic shock are an extremely high risk group. Attempted PCI in this setting must be considered extremely carefully, because it seems probable that the natural course of the condition will not be altered. Every step should be taken to reduce the incidence of shock in these patients (reducing time to treatment and use of primary PCI in preference to thrombolysis).

Secondly, we have found that outcome is related to age, as did the SHOCK trial, with patients over 70 years faring far worse than younger patients. However, with a relatively small cohort of patients, it is difficult to know for certain whether there is no benefit for the elderly or beyond which age the likelihood of harm exceeds that of benefit. Many elderly patients in the SHOCK registry fared well with an early invasive strategy, ${ }^{21}$ and Menon and Hochman ${ }^{22}$ suggested that a selective approach to an invasive strategy for elderly patients is probably more appropriate than not offering this treatment.
Thirdly, patients with cardiogenic shock complicating failure to reperfuse are a higher risk group than those undergoing primary angioplasty for cardiogenic shock or angioplasty for cardiogenic shock complicating a reinfarction. Whether this relates to delay, extent of myocardial injury, failure to change the natural history in a high risk group, or some adverse effect of the combination of thrombolytic treatment and PCI is unknown.

As cardiogenic shock remains a problem associated with adverse outcome, attempts to reduce its incidence are to be encouraged. It is possible that strict risk stratification of patients after an initial episode of AMI and revascularisation for high risk patients at that stage will result in patients tolerating second events better. For all patients with AMI, early presentation to hospital, early diagnosis, prompt initiation of reperfusion therapy, and early liaison with a revascularisation unit at the first signs of haemodynamic instability are essential components in reducing the incidence of and managing cardiogenic shock. The CAPTIM (comparison of angioplasty and pre-hospital thrombolysis in acute myocardial infarction) study group had a lower incidence of cardiogenic shock in the pre-hospital thrombolysis arm than in the primary PCI arm..$^{23}$ These data are considered by some to indicate superiority of this reperfusion strategy. Nonetheless, rapid identification, pick up, and transfer to appropriate revascularisation centres of patients with AMI are also likely to reduce the incidence of cardiogenic shock.

It is of interest that we did not find clear mortality differences between those receiving revascularisation within a relatively short period of time from the onset of major symptoms and those undergoing revascularisation much later. Although the numbers of patients are small, patients undergoing revascularisation within six hours of symptom onset fared slightly better than those undergoing intervention 6-12 hours after symptom onset. Patients undergoing intervention later than this who survive are possibly self selected survivors, but the SHOCK data do suggest that PCI for cardiogenic shock should be considered beyond the traditional 12 hour window after the onset of symptoms.

For patients with adverse outcomes despite attempted emergency PCI with IABP support, alternative strategies must be considered. These include earlier use of IABP before transfer; transfer by air ambulance; better PCI with thrombus extraction devices; the use of left ventricular assist devices; cooling; further investigation of the role of nitric oxide synthase inhibitors ${ }^{24}$; ; and intubation and ventilation with subsequent management of these patients by dedicated intensivists in a cardiac intensive unit setting.

\section{Study limitations}

Data for some patients are incomplete. In addition, the severity of these patients' conditions is the cause for failure to obtain data that traditionally have been required to diagnose cardiogenic shock. Our patients were too sick for us to contemplate measurement of filling pressures or calculation of cardiac output and urine output before performing angiography and attempted revascularisation. However, we are satisfied on clinical grounds that cardiogenic shock was present in each instance.

We have been unable to collect the data on the onset of cardiogenic shock to intervention times, as these data are often not available from the hospital case notes.

The cohort does not include patients with clear evidence of a mechanical problem at initial assessment, nor does it include patients for whom the initial revascularisation strategy was CABG. Therefore, the cohort does not reflect the complete spectrum of patients presenting with cardiogenic shock complicating AMI. This is in part because of the design of the study, which was set up primarily to study 
patients undergoing PCI for cardiogenic shock, but, as with all retrospective analyses, we do not know the selection criteria to which these patients were subjected before being referred. Selection bias also exists for randomised studies. In the SHOCK trial, the 30 day mortality for all patients randomised into the trial was significantly lower than for non-randomised patients in the registry with cardiogenic shock not due to mechanical causes. ${ }^{10}$

In the absence of routine echocardiography and left ventriculography before PCI, some patients with mechanical problems best treated surgically may have been missed, but we think the likelihood of this is low. In fact, just two of our patients developed a ventriculoseptal defect and both died. These patients were included in the analysis. All surviving patients underwent echocardiography after PCI.

\section{Conclusions}

This is a contemporary experience of the management of cardiogenic shock, in which many of the principles of the SHOCK trial were applied. Our experience supports the view that emergency angiography and attempted revascularisation in conjunction with intensive haemodynamic support is a strategy that saves the lives of patients with cardiogenic shock by comparison with conventional medical management. However, this strategy is more likely to be successful in some groups of patients than in others. For some patients, progression to an adverse outcome is not altered by attempted emergency revascularisation. In fact, an adverse outcome is probably determined well before emergency revascularisation can even be contemplated. This has implications for the transfer of acutely ill patients from a district general hospital to a regional cardiothoracic unit. Patients of advanced age, with a history of myocardial infarction, with shock in the context of failed thrombolysis, or with previous CABG should only be transferred urgently for attempted revascularisation after serious consideration of the low chance of a successful outcome and detailed discussion with the patient and family members. The results for patients with none of these features strongly support the policy of early interventional management.

Every effort should be made to reduce the incidence of cardiogenic shock. For patients who do badly despite conventional emergency revascularisation measures, further investigation into the role of alternative treatments is required. Finally, audit of cardiogenic shock management and outcome is essential, taking into account that failure to change the natural history of the condition is not the same as a complication of treatment.

\section{Authors' affiliations}

A G C Sutton, J A Hall, A A Harcombe, R A Wright, M A de Belder, Cardiothoracic Division, The James Cook University Hospital, Middlesbrough, UK

P Finn, Postgraduate Institute, School of Health and Social Care, University of Teesside, Middlesbrough, UK

\section{REFERENCES}

1 Goldberg RJ, Gore JM, Alpert JS, et al. Cardiogenic shock after acute myocardial infarction: incidence and mortality from a community-wide perspective, 1975 to 1988 . N Engl J Med 1991;325:1117-22.
2 Holmes DRJ, Bates ER, Kleiman NS, et al. Contemporary reperfusion therapy for cardiogenic shock: the GUSTO-I trial experience. The GUSTO-I investigators. Global utilization of streptokinase and tissue plasminogen activator for occluded coronary arteries. J Am Coll Cardiol 1995;26:668-74.

3 GISSI Investigators. Effectiveness of intravenous thrombolytic treatment in acute myocardial infarction. Gruppo Italiano per lo studio della streptochinasi nell'nfarto miocardico (GISSI). Lancet 1986;i:397-402.

4 Lee L, Bates ER, Pitt B, et al. Percutaneous transluminal coronary angioplasty improves survival in acute myocardial infarction complicated by cardiogenic shock. Circulation 1988;78:1345-51.

5 Hibbard MD, Holmes DRJ, Bailey KR, et al. Percutaneous transluminal coronary angioplasty in patients with cardiogenic shock. J Am Coll Cardiol 1992; 19:639-46.

6 Moosvi AR, Khaja F, Villanueva L, et al. Early revascularization improves survival in cardiogenic shock complicating acute myocardial infarction. J Am Coll Cardiol 1992;19:907-14.

7 Gacioch GM, Ellis SG, Lee L, et al. Cardiogenic shock complicating acute myocardial infarction: the use of coronary angioplasty and the integration of the new support devices into patient management. J Am Coll Cardiol 1992; 19:647-53

8 Eltchaninoff $\mathrm{H}$, Simpfendorfer $\mathrm{C}$, Franco $\mathrm{I}$, et al. Early and 1 -year survival rates in acute myocardial infarction complicated by cardiogenic shock: a retrospective study comparing coronary angioplasty with medical treatment. Am Heart J 1995; 130:459-64.

9 Berger PB, Holmes DR, Stebbins AL, et al. Impact of an aggressive invasive catheterization and revascularization strategy on mortality in patients with cardiogenic shock in the global utilization of streptokinase and tissue plasminogen activator for occluded coronary arteries (GUSTO-I) trial: an observational study. Circulation 1997:96:122-7.

10 Hochman JS, Sleeper LA, Webb JG, et al. Early revascularization in acute myocardial infarction complicated by cardiogenic shock. SHOCK investigators. Should we emergently revascularize occluded coronaries for cardiogenic shock. N Engl J Med 1999:341:625-34.

11 Price DJA, Campbell PG, Sutton AGC, et al. Selective use of abciximab in coronary stenting: overall outcomes can still be equivalent to those in the EPISTENT treatment group. Int J Cardiovasc Interv 2001;4:15-20.

12 The TIMI Study Group. The thrombolysis in myocardial infarction (TIMI) trial: phase 1 findings. N Engl J Med 1985;312:932-6.

13 The GUSTO Investigators. An international randomized trial comparing four thrombolytic strategies for acute myocardial infarction. N Engl J Med 1993;329:673-82.

14 Hochman JS. Cardiogenic shock complicating acute myocardial infarction: expanding the paradigm. Circulation 2003;107:2998-3002.

15 Ryan TJ. Early revascularization in cardiogenic shock - a positive view of a negative trial. N Engl J Med 2003;341:687-8.

16 Hochman JS, Sleeper LA, White HD, et al. One-year survival following early revascularization for cardiogenic shock. JAMA 2001;285:190-2.

17 Webb JG, Lowe AM, Sanborn TA, et al. Percutaneous coronary intervention for cardiogenic shock in the SHOCK trial. J Am Coll Cardiol 2003:42:1380-6.

18 Webb JG, Sanborn TA, Sleeper LA, et al. Percutaneous coronary intervention for cardiogenic shock in the SHOCK Trial Registry. Am Heart $J$ $2001 ; 141: 964-70$

19 Sanborn TA, Sleeper LA, Bates ER, et al. Impact of thrombolysis, intra-aortic balloon pump counterpulsation, and their combination in cardiogenic shock complicating acute myocardial infarction: a report from the SHOCK trial registry. Should we emergently revascularize occluded coronaries for cardiogenic shock? J Am Coll Cardiol 2000;36:1123-9.

20 Sutton AGC, Campbell PG, Price DJA, et al. Failure of thrombolysis by streptokinase: detection with a simple electrocardiographic method. Heart 2000:84:149-56.

21 Dzavik V, Sleeper LA, Cocke TP, et al. Early revascularization is associated with improved survival in elderly patients with acute myocardial infarction complicated by cardiogenic shock: a report from the SHOCK trial registry. Eur Heart J 2003;24:828-37.

22 Menon V, Hochman JS. Management of cardiogenic shock complicating acute myocardial infarction. Heart 2002;88:531-7.

23 Bonnefoy E, Lapostolle F, Leizorovicz A, et al. Primary angioplasty versus prehospital fibrinolysis in acute myocardial infarction: a randomised study. Lancet 2002:360:825-9.

24 Cotter G, Kaluski E, Blatt A, et al. L-NMMA (a nitric oxide synthase inhibitor) is effective in the treatment of cardiogenic shock. Circulation 2000;101:1358-61.

25 Cotter G, Kaluski E, Milo O, et al. LINCS: L-NAME (a NO synthase inhibitor) in the treatment of refractory cardiogenic shock. Eur Heart $J$ 2003;24:1287-95. 